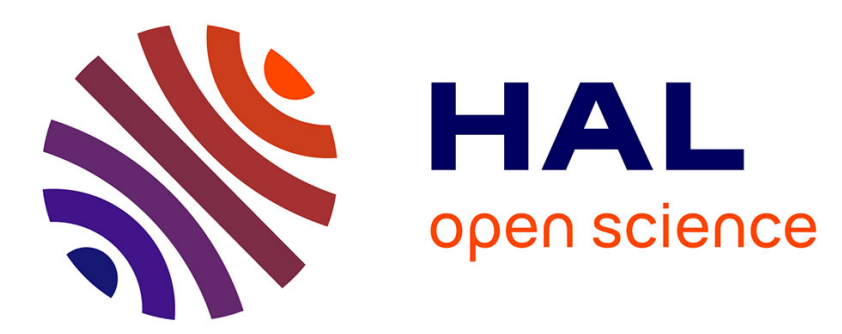

\title{
Segmental order in a uniaxially constrained polydimethylsiloxane network: a deuterium magnetic resonance study
}

B. Deloche, M. Beltzung, J. Herz

\section{- To cite this version:}

B. Deloche, M. Beltzung, J. Herz. Segmental order in a uniaxially constrained polydimethylsiloxane network: a deuterium magnetic resonance study. Journal de Physique Lettres, 1982, 43 (22), pp.763769. 10.1051/jphyslet:019820043022076300 . jpa-00232123

HAL Id: jpa-00232123

https://hal.science/jpa-00232123

Submitted on 1 Jan 1982

HAL is a multi-disciplinary open access archive for the deposit and dissemination of scientific research documents, whether they are published or not. The documents may come from teaching and research institutions in France or abroad, or from public or private research centers.
L'archive ouverte pluridisciplinaire HAL, est destinée au dépôt et à la diffusion de documents scientifiques de niveau recherche, publiés ou non, émanant des établissements d'enseignement et de recherche français ou étrangers, des laboratoires publics ou privés. 
LE JOURNAL DE PHYSIQUE-LETTRES

J. Physique - LETTRES 43 (1982) L-763 - L-769

15 NOVEMBRE 1982, PAGE L-763

Classification

Physics Abstracts

$61.16 \mathrm{~N}-61.40 \mathrm{~K}$

\title{
Segmental order in a uniaxially constrained polydimethylsiloxane network : a deuterium magnetic resonance study $\left({ }^{*}\right)\left({ }^{* *}\right)$
}

\author{
B. Deloche
}

Laboratoire de Physique des Solides (***), Bât. 510, Université de Paris-Sud, 91405 Orsay, France

M. Beltzung and J. Herz

Centre de Recherches sur les Macromolécules, 6, rue Boussingault, 67083 Strasbourg, France

(Reçu le 8 juillet 1982, accepté le 30 septembre 1982)

Résumé - L'ordre orientationnel induit dans un réseau élastique sous contrainte uniaxiale est étudié par RMN du deutérium. Les spectres de chaînes perdeutérées d'un réseau modèle de polydiméthylsiloxane sous tension ou compression présentent un doublet quadrupolaire. Des expériences pour différentes orientations de la contrainte par rapport au champ magnétique du spectromètre montrent qu'une anisotropie uniaxiale est mesurée au niveau des segments du polymère. De plus l'écartement du doublet a une dépendance explicite en fonction du taux d'élongation.

\begin{abstract}
The orientational order generated in a cross-linked rubber by a uniaxial stress is probed with deuterium NMR. Spectra of perdeuterated polymer chains of a polydimethylsiloxane model network exhibit quadrupolar doublets under both elongation and compression. Experiments for various orientations of the stress relative to the spectrometer magnetic field show that an uniaxial anisotropy is induced at the level of the chain segments. The doublet splitting has an explicit dependence on the extension ratio.
\end{abstract}

1. Introduction - The studies of elastic and opticoelastic properties of cross-linked rubbers have revealed some deviations from the classical kinetic theory which have been ascribed to correlations between chain segments in the deformed network [1]. This is one of the reason why various experimental techniques sensitive to the local behaviour are currently being used to study elastomeric chains under deformation. For instance orientational correlations have been studied by fluorescent polarization [2] and by deuteron magnetic resonance $\left({ }^{2} \mathrm{H}\right.$ NMR) using deuterated swelling agents [3]. The common feature of both approaches is to use additional small molecules

(*) This work has been presented at the Macromolecular Symposium of the International Union of Pure and Applied Chemistry (IUPAC), July 1982, Amherst, Massachusetts (U.S.A.).

$\left({ }^{* *}\right)$ Part of this work has been done with the financial support of the DRET (Contract No 80665).

$\left({ }^{* * *}\right)$ Laboratoire associé au C.N.R.S. 
to probe the local orientational order generated in the deformed network : specifically ${ }^{2} \mathrm{H} \mathrm{NMR}$ experiments have shown that solvent molecules exhibit a non-zero order parameter which is very sensitive to the sample elongation. However this kind of investigation is inherently limited because it provides an indirect measure of the order of the chain segments and, in addition, the probe itself may locally perturb the system especially in the case of fluorescence. Obviously the relevant method to obtain specific information on the local anisotropy would be to directly observe the polymer chains. We know from studies on various systems of deuterated liquid crystals $[4,5]$ that the ${ }^{2} \mathrm{H}$ NMR technique is well adapted to such a purpose. We propose here to monitor the chain behaviour, extending the ${ }^{2} \mathrm{H}$ NMR experiments to deuterated units of the network chains. Specifically we report in this letter the first direct measurements of chain segment orientation performed on a dry deuterated polydimethylsiloxane network in an uniaxial stress for both elongation and compression.

2. Experimental. - 2.1 SAMPLeS. - Experiments were performed on a well defined polydimethylsiloxane network with a known fraction $(\sim 20 \%)$ of perdeuterated chains

$$
\left.+\mathrm{Si}\left(\mathrm{CD}_{3}\right)_{2}-\mathrm{O}\right)_{n} \text {, }
$$

PDMS(D). This was synthetized by reacting stoichiometric mixtures of labelled and unlabelled precursors chains of $\alpha, \omega$ di(hydrogeno)polydimethylsiloxane with tetra(allyloxy)ethane, as a tetrafunctional cross-link [6]; the cross or " endlinking " reaction was carried out in toluene at $70 \%$ polymer concentration. The synthesis of perdeuterated precursors is described in reference [7]. For the present investigation the average molecular weight between cross-links is 25000 (the number of monomer/network chains is roughly 350). The molecular weight distribution is about 1.5 , the same as for the precursor polymer.

2.2 UNIAXIAL STRESS. - Tension was applied to the sample $(20 \mathrm{~mm} \times 5 \mathrm{~mm} \times 0.8 \mathrm{~mm})$ as described in reference [3] so that the stretching direction is coincident with the axis of the NMR tube. The tension was either parallel or at right angles to the spectrometer magnetic field $\mathbf{B}_{0}$. The extension ratio $\lambda=L / L_{0}$ was estimated from the stretched and unstretched lengths measured between marks on the sample; $L$ was determined to within $\pm 0.2 \mathrm{~mm}$.

The compression was obtained by squeezing a small piece of the sample $(10 \mathrm{~mm} \times 3 \mathrm{~mm} \times$ $0.8 \mathrm{~mm}$ ) between two semi-cylindrical jaws made of KELF and lubricated with silicone oil. This assembly was introduced into the bottom of the NMR tube; in this way the angle $\Omega$ between the compression direction relative to $\mathbf{B}_{0}$ can be varied continuously by rotating the tube by hand around its vertical axis, as indicated on figure 1 . The compression ratio $(\lambda<1)$ was estimated roughly from the thickness of the sample.

2.3 NMR EQUIPMENT. - ${ }^{2} \mathrm{H}$ NMR spectra were obtained after signal averaging (because of the inherently poor filling factor in the $10 \mathrm{~mm}$ NMR tube) from two FT NMR equipments : a C X P 90 Bruker spectrometer operating at $2.1 \mathrm{~T}$ with $\Omega=90^{\circ}$ and the spectrometer of the Biophysics Laboratory (Ecole Polytechnique) operating at $6.4 \mathrm{~T}$ with $\Omega=0^{\circ}$ (see Fig. 1).

3. Results - Figure 2 shows the change in the ${ }^{2} \mathrm{H}$ NMR spectrum of PDMS(D) from a single line $(\lambda=1$, relaxed state) to a quadrupolar doublet as the sample is uniaxially deformed along a direction perpendicular to the steady magnetic field $\mathbf{B}_{0}\left(\Omega=90^{\circ}\right)$. The finite values of the observed splitting $\Delta v$ are indicative of orientational anisotropy of the $\mathrm{Si}-\mathrm{CD}_{3}$ bonds. In addition the observed lineshapes also exhibited wings whose origin is not yet well understood; specifically for $\lambda=1$ their contribution is larger $(\sim 20 \%)$ than that of a Lorentzian line.

A doublet is still observed when the sample is stretched along a direction parallel to $\mathbf{B}_{0}\left(\Omega=0^{\circ}\right)$, but the $\Delta v$ are exactly double the previous case $\left(\Omega=90^{\circ}\right)$ for the same $\lambda$. Moreover an angular dependence $\Delta v(\Omega)$ is also observed when the direction of the uniaxial compression with respect to 


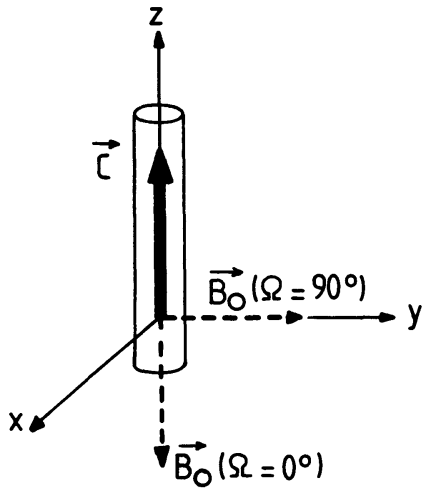

a) tension

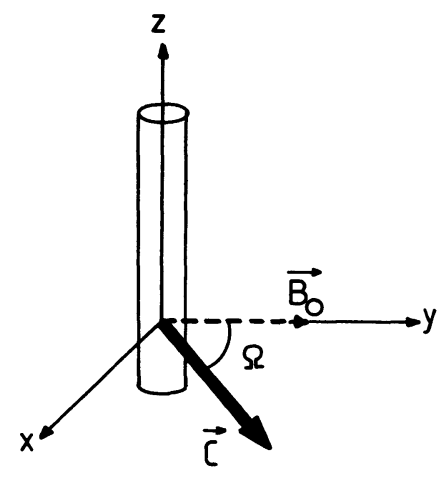

b) compression

Fig. 1. - Orientation of the spectrometer magnetic field $\mathbf{B}_{0}$ with respect to the applied uniaxial stress $\mathbf{C}$ : a) $\mathbf{B}_{0}$ is along the $y$ or the $z$ axis, i.e. perpendicular or parallel to the direction of the elongation, coincident with the NMR tube axis; $b$ ) $\mathbf{B}_{0}$ is always along the $y$ axis while the direction of the compression is varied within the plane $(x, y)$, by rotating the NMR tube around its vertical axis.

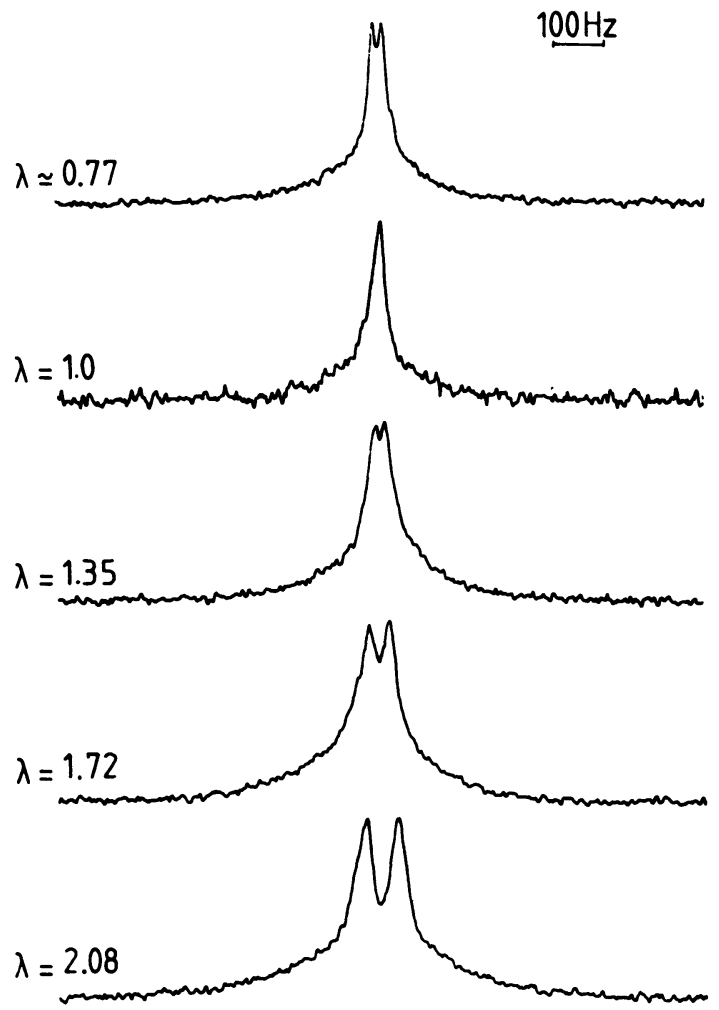

Fig. 2. - $13 \mathrm{MHz}^{2} \mathrm{H}$ NMR spectra of perdeuterated chains of PDMS network as the sample is uniaxially compressed $(\lambda<1)$ or elongated $(\lambda>1)$ along a direction perpendicular to the steady magnetic field $\left(\Omega=90^{\circ}\right)$. The number of accumulation was typically 5 for $\lambda=1.0$ and 250 for $\lambda=2.08$. 
$\mathbf{B}_{0}$ is changed : a magic angle narrowing $(\Delta v \sim 0)$ occurs for $\Omega \sim 55^{\circ}$, whereas $\Delta v$ is increased by a factor two for $\Omega=0^{\circ}$, similar to the case of elongation pointed out above. Finally the complete angular variation $\Delta v(\Omega)$ reported in figure 3 shows that it reproduces exactly $\left|P_{2}(\cos \Omega)\right|$, as in relation (3) of the appendix.

Figure 4 illustrates that $\Delta v$ is a linear function of $\lambda^{2}-\lambda^{-1}$ for $\lambda>1$. Because of the breaking tensile strength this investigation was limited to extension ratios lower than 2.5 .

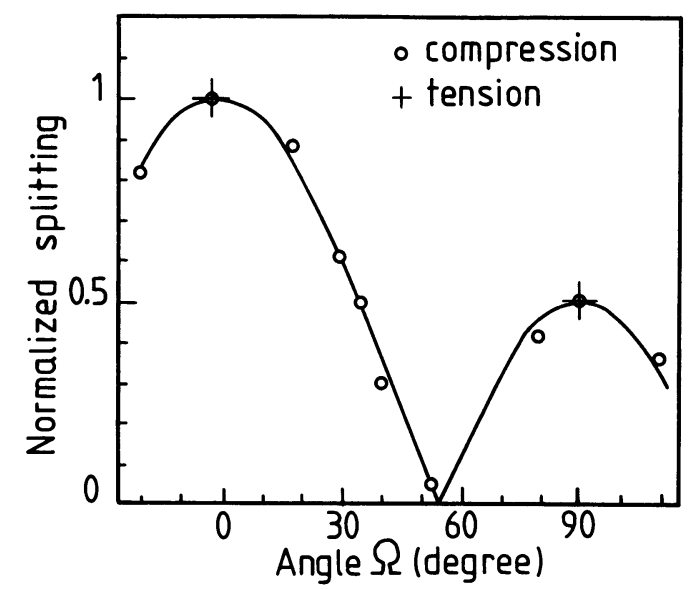

Fig. 3. - Variation of the normalized splittings as a function of the angle between the applied uniaxial constraint (compression or tension) and the steady magnetic field. The continuous line is the function $\left|P_{2}(\cos \Omega)\right|$.

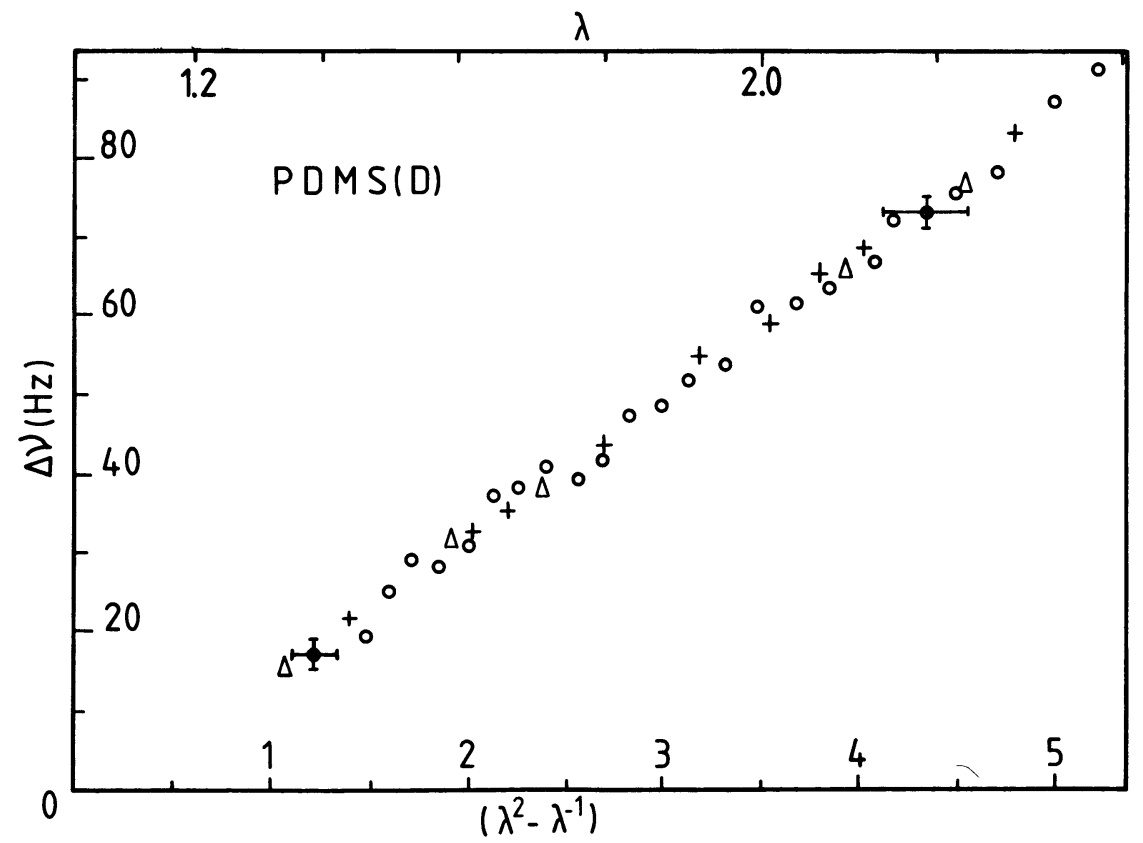

Fig. 4. - Quadrupolar splittings $\Delta v$, observed in the perpendicular geometry $\left(\Omega=90^{\circ}\right), v s . \lambda^{2}-\lambda^{-1}$. The data correspond to different experiments with various pieces of sample. The values of $\Delta v$ are reversible with the extension ratio $\lambda$. 
4. Discussion. - In the rubbery state exhibited at room temperature by various polymers, the chains, held by cross-link junctions, are fluid whereas the bulk material retains its form. This fluidity (high frequency motions of monomers units) which assures the liquid-like character of these compounds is particularly pronounced in the case of the PDMS : indeed its glass transition temperature is low $\left(T_{\mathrm{g}} \sim-120^{\circ} \mathrm{C}\right)$ and its chain backbone has minimal barriers to internal rotations. In addition, from the ${ }^{2} \mathrm{H}$ NMR point of view, the fact that we are dealing with only methyl deuterons limits the linewidth at half height in the relaxed state [8] and thus makes the experiment more sensitive to changes when the system is uniaxially deformed.

The appearance of a doublet spectrum for $\lambda \neq 1$, in conjunction with the angular dependence of $\Delta v(\Omega)$ (Fig. 3), demonstrates that this experiment allows a uniaxial anisotropy at the level of chain segments to be detected. Spectral wings also spread out with increasing $\lambda$ and might be assigned to deuterons whose dynamical behaviour is not necessarily the same as that of deuterons associated with the doublet. This aspect has not, however, been specifically studied in this work and so will not be considered here.

The doublet corresponds to deuterons of segments whose rapid orientational motions around the direction of the applied tension or compression do not average the quadrupolar coupling to zero. As indicated in the appendix, a residual interaction is observed along the stress axis which is a symmetry axis for frequencies smaller than the segmental motion frequencies which contribute to the averaging process ; the lower limit of the latter may be given by the frequency of the modulated interaction i.e. about $10^{5} \mathrm{~s}^{-1}$. Thus, for such a soft rubber network, the polymer chains (or a portion of them) which give rise to the doublet may be visualized as a uniaxial fluid of segments exhibiting anisotropic reorientational diffusion with respect to the direction of the applied constraint. For instance we may consider that the chain segments diffuse in such a way that they cannot be oriented in any other direction than along the axis of stress for times longer than a few $10^{-5} \mathrm{~s}$; obviously this scheme is not exclusive. Another striking feature of the averaging process is that the spectra are composed of a single well defined doublet. This implies that, within the linewidth limit of this structure, the methyl groups are equivalent on the time scale of the ${ }^{2} \mathrm{H}$ NMR and that the degree of segmental orientation is quasi uniform among the various polymer chains (or portions of them) and along their lengths. The induced orientational order parameter $\left\langle P_{2}(\cos \theta)\right\rangle$ of the $\mathrm{Si}-\mathrm{CD}_{3}$ bonds can be deduced from relation (3) of the appendix taking into account the reduction factor due to random rotation of the methyl group around its threefold axis and assuming the quadrupolar constant $v_{\mathrm{q}}$ is close to $176 \mathrm{kHz}$ for this type of deuteron [9]; $\left\langle P_{2}(\cos \theta)\right\rangle$ is found to be $1.26 \times 10^{-3}$ for $\lambda=2$.

Finally the characteristic $\lambda$-dependence of the orientational order (Fig. 4) is found to be identical to that previously observed on polyisoprene networks using deuterated swelling agents [3]. A phenomenological analysis has shown that this chain segment behaviour is related to the isovolume and affine characters of the orientation process [3]. Also various statistical treatments, based on models of gaussian networks with " affine junctions ", have led to the same $\lambda$-dependence [10-13] and to the determination of segmental orientation functions [12]. Comparisons of these theoretical results with our experiments in conjunction with recent neutron scattering investigation on the affine behaviour of the same network [14] are currently in progress [15].

5. Concluding remarks. - The results reported in this work show that the ${ }^{2} \mathrm{H}$ NMR technique applied to labelled polymeric chains is a powerful tool for probing segmental ordering generated in uniaxially constrained rubber networks. This technique would have to be extended to deuterium labels at specific sites on the network chains to obtain a more precise picture of local changes in the elastomer. In addition, the short range orientational behaviour of the segments derived from these preliminary ${ }^{2} \mathrm{H}$ NMR experiments complements the long range configurational behaviour deduced from recent neutron scattering experiments $[7,14]$ for a complete description of the response of the PDMS network to external stress. 
Acknowledgments. - One of us, B. D., is indebted to the Biophysics Laboratory (Laboratory of Prof. M. Guéron, Ecole Polytechnique) for use of the NMR equipment : the assistance of Drs. J. L. Leroy and P. Plateau during the experiments was greatly appreciated. B. D. is also grateful to Prof. M. T. Shaw (Storrs, Connecticut) for useful discussions before the beginning of this work relative to the application of the compression.

Appendix. - Because of the tensorial coupling of the deuteron quadrupolar moment with the electric field gradient (e.f.g.) of the charge distribution of the C-D bond, the conventional ${ }^{2} \mathbf{H}$ NMR line is split into a symmetrical doublet [16]. With an axial e.f.g. tensor, the doublet spacing is given in frequency units by :

$$
\Delta v=\frac{3}{2} v_{4}\left(P_{2}(\cos \Theta)\right)
$$

$v_{\mathrm{q}}$ is the static quadrupolar coupling constant. The $\boldsymbol{P}_{2}$ term is the second Legendre polynomial; $\Theta$ is the angle between the C-D bond and the direction of the steady magnetic field $B_{0}$. There is one such quadrupolar doublet for each chemical site and for each distinct orientation of the C-D bond relative to $B_{0}$. For a static distribution of C-D bond vectors, the ${ }^{2} \mathrm{H} N M R$ spectra consist of characteristic powder spectra $[17,18]$.

Motions of the C-D bond modulate $\Theta$ and a time average has to be taken when the C-D bond reorientates with frequencies larger than that of the static interaction ( $200 \mathrm{kHz})$; relation (1) then becomes :

$$
\Delta v=\frac{3}{2} v_{\mathrm{q}}\left\langle P_{2}(\cos \Theta(t))\right\rangle .
$$

So the quadrupolar splitting appears as proportional to the orientational order parameter of the C-D bond with respect to $B_{0}$. Because of such averagings, the ${ }^{2} H$ NMR spectrum of an anisotropic fluid medium consists of doublets that can be discrete and well resolved [4].

In the case of uniaxial reorientations around a symmetry axis (a macroscopic one for instance) making the angles $\Omega$ with $B_{0}$ and $\theta$ with the C-D bond, the averaging over azimuthal angles leads to the following expression for the splitting :

$$
\Delta v=\frac{3}{2} v_{\mathrm{G}}\left\langle P_{2}(\cos \theta(t))\right\rangle P_{2}(\cos \Omega) .
$$

Then the effective quadrupolar coupling constant is $v_{\mathrm{q}}\left\langle\boldsymbol{P}_{\mathbf{2}}(\cos \theta(t))\right\rangle$ where $\left\langle\boldsymbol{P}_{\mathbf{2}}(\cos \theta(t))\right\rangle$ can be defined as the orientational order parameter of the C-D bond relative to the symmetry axis.

In the particular case of methyl deuterons, an additional averaging process has to be taken into account due to the random rotation of the $\mathrm{CD}_{3}$ groups around their threefold axis : $\Delta v$ is then proportional to the degree of orientational order of the methyl symmetry axis.

\section{References}

[1] See for instance :

Treloar, L. R. G., The Physics of Rubber Elasticity (Clarendon Press, Oxford) 1975, Chapter 9 and references cited therein.

StEIN, R. S., Rubber Chem. Technol. 49 (1976) 458 and references cited therein.

[2] Jarry, J. P. and Monnerie, L., J. Polym. Sci. 18 (1980) 1879.

[3] Deloche, B. and SAMUlSKI, E. T., Macromolecules 14 (1981) 575.

[4] Charvolin, J. and Deloche, B., The Molecular Physics of Liquid Crystals, ed. by G. R. Luckhurst and G. W. Gray (Academic Press, London) 1979, Chapter 15 and references cited therein.

[5] Czarniecka, K. and Samulski, E. T., Mol. Cryst. Liq. Cryst. 63 (1981) 205.

[6] Herz, J., Belkebir, A. and Rempr, P., Eur. Polym. J. 9 (1973) 1165.

[7] Beltzung, M., Picot, C., RempP, P. and Herz, J., in press (Macromolecules). 
[8] The precise nature of the line is not clear; we anticipate that the effects observed by COHEN-ADDAD, J. P. et al. (J. Chem. Phys. 76 (1982) 2744) on the same kind of networks with proton NMR could contribute to the linewidth. However this has no influence on the conclusions of this work.

[9] Ader, A. and Loewenstein, A., J. Am. Chem. Soc. 96 (1974) 5336.

[10] Roe, R. J. and Krigbaum, W. R., J. Appl. Phys. 35 (1964) 2215.

[11] Tanaka, T. and Allen, G., Macromolecules 10 (1977) 426.

[12] Ullman, R., Macromolecules 11 (1978) 987.

[13] JARRY, J. P. and MONNERIE, L., Macromolecules 12 (1979) 316.

[14] Beltzung, M., Herz, J., Picot, C., Bastide, J. and Duplessix, R., Proceedings of the 27th International Symposium on Macromolecules (Strasbourg) 2 (1981) 728.

[15] For instance the treatment of our data in the framework of the R. Ullman's analysis [12] allow us to estimate directly the reduced variable $\omega$ which is related to the number of segments which move cooperatively in the orientation process and the number of statistical segments.

[16] Cohen, M. H. and Reiff, F., Solid State Phys. 5 (1975) 321, ed. by F. Seitz and D. Turnbull. Also, see the background section of [3] and references cited therein.

[17] HentsChel, D., SillesCu, H. and SPiess, H. W., Polymer 22 (1981) 1516.

[18] Meurer, B., Spegt, P. and Weill, G., Proceedings of the 27th International Symposium on Macromolecules (Strasbourg) 2 (1981) 864. 